\title{
Meta
}

Journal des traducteurs

Translators' Journal

\section{RÉZEAU, Pierre (1993) : Petit dictionnaire des chiffres en toutes \\ lettres, Paris, Seuil, 254 p.}

\section{META}

\section{Pierre Rézeau}

Volume 39, numéro 3, septembre 1994

URI : https://id.erudit.org/iderudit/003947ar

DOI : https://doi.org/10.7202/003947ar

Aller au sommaire du numéro

Éditeur(s)

Les Presses de l'Université de Montréal

ISSN

0026-0452 (imprimé)

1492-1421 (numérique)

Découvrir la revue

Citer ce compte rendu

Rézeau, P. (1994). Compte rendu de [RÉZEAU, Pierre (1993) : Petit dictionnaire des chiffres en toutes lettres, Paris, Seuil, 254 p.] Meta, 39(3), 492-497.

https://doi.org/10.7202/003947ar

Ce document est protégé par la loi sur le droit d'auteur. L'utilisation des services d'Érudit (y compris la reproduction) est assujettie à sa politique d'utilisation que vous pouvez consulter en ligne.

https://apropos.erudit.org/fr/usagers/politique-dutilisation/
Cet article est diffusé et préservé par Érudit.

Érudit est un consortium interuniversitaire sans but lucratif composé de l’Université de Montréal, l'Université Laval et l'Université du Québec à Montréal. Il a pour mission la promotion et la valorisation de la recherche. https://www.erudit.org/fr/ 
RÉZEAU, Pierre (199) ): Petit dictionnaire des chiffres en toutes lettres, Paris, Seuil, $254 \mathrm{p}$.

\section{«À moi, comte, deux mots !»}

On a voulu rassembler ici les faits de lexique contemporains dans lesquels les nombres n'ont pas leur valeur arithmétique (ainsi trois dans il pleut trois gouttes, cinq dans j'en ai pour cinq minutes, trente-six dans voir trente-six chandelles).

De tels emplois ne sont pas nés d'hier et les dictionnaires en ont déjà recensé un certain nombre, mais les présenter en tir groupé est riche d'enseignements: examiner ainsi un sous-ensemble de la langue permet d'en faire le tour sans rien omettre d'essentiel et de dresser une nomenclature systématique. On peut aussi, chemin faisant, enregistrer de nombreuses variantes, tantôt parfaitement lexicalisées mais vieillies ou moins usuelles, tantôt ponctuelles et quelquefois fantaisistes, mais qui dans tous les cas témoignent de la santé et de l'originalité du français (les quelques first class, number one et autres fiftyfifty qu'on y rencontre relèvent plus du pied de nez malicieux que des derniers outrages à notre langue).

Pour faire bonne mesure, on a cependant inséré au fil de l'ouvrage quelques mots ou expressions qui n'ont pas de valeur figurée ni métaphorique, et dans lesquelles les nombres ont leur valeur arithmétique (ainsi compter jusqu'à trois, faire ses trois jours ou encore cinq, quatre, trois, deux, un, partez!), tout simplement parce que ces tours font défaut dans les dictionnaires ou y sont mal représentés.

Il était tentant d'accompagner chemin faisant ces relevés de slogans (Les Trois Suisses se mettent en quatre), de titres de livres (Zéro pour la question, de San Antonio) 
ou de films (Les 400 coups, de François Truffaut), têmoignant à leur manière de la vitalité de cet aspect de la langue ou y ayant contribué. On y a cependant renoncé, dans la mesure où leur abondance aurait quasiment doublé le travail.

\section{«Ch. à louer 2 p. cuisine, proximité piscine» \\ On n'a donc pas retenu ici :}

- les mots ou expressions bien attestés, dans lesquels on a affaire à une simple valeur arithmétique : né d' un premier lit, un deux-pièces (vêtement ou logement), un trois-mâts (bateau), un cent mètres (course), un trois-quarts (joueur de rugby, manteau ou violon), etc.;

- les chiffres renvoyant à des objets manufacturés: un [pistolet de calibre] 7,65 ou une 15 [CV Citroën]; aux degrés d'une échelle : alcool à $90^{\circ}$, fil-en-trois (fil-en-quatre, etc.); à une division quelconque: le fameux article 49/3 de la Constitution de la Ve République; et, d'une manière générale, les chiffres entrant dans des listes, ainsi un 75 pour désigner un conducteur dont le véhicule est immatriculé dans la Seine;

- dans le cas de un, il était normal de ne pas prendre en compte les locutions elliptiques dans lesquelles le mot correspond à l'article indéfini et non à l'article numéral, mais on a taillé une cote plutôt large dans quelques cas où il eût été un peu précipité de trancher.

\section{O 201000} ludique:

On a également écarté les expressions qui appartiennent exclusivement au domaine

- Plaisanteries, ponctuelles du type: «charmante jeune femme [...] à qui sa taille exiguë avait valu le sobriquet de la môme Zéro-Virgule-Cinq» (A. Allais, Euvres anthumes) ou très connues comme: «[...] 'Quand t'as été à Moulins, sûr que t'as été aux Six-Fesses.' On avait ainsi baptisé un bistrot de Moulins tenu par la mère et les deux filles, toutes allègres du séant $[. .$.$] » (R. Fallet, Un idiot à Paris) ou encore : «Quand, en voiture, nous arrivions$ en vue de Cormonville, ma mère, me montrant la haute flèche du clocher entourée de ses quatre clochetons, avait coutume de me lancer: ' $\grave{A}$ Cormonville sont cinq clochers et quatre sans cloches.' Et moi, depuis longtemps, j'avais éventé le mystère de ce calembour en forme de dicton et ces quatre cents cloches ne me faisaient plus aucun effet.» (C. Menuet, Une enfance ordinaire);

- rébus, du type : «[...] Ker-Fleurette et 7-A-C, deux odieuses bicoques [...].» (H. Bazin, La mort du petit cheval) ou bien «[...] la fin du bistrot $\mathrm{O} 20100 \mathrm{O}$, 'Au vin sans eau !', fini tout ça!» (R. Sabatier, Boulevard);

devinettes, comme «20-100 000 ânes dans un pré et 120 dans l'autre [Vincent mit l'âne dans un pré et s'en vint dans l'autre]. Combien y a-t-il en tout de pattes et d'oreilles ?» (cf. M. Rouanet, Nous les filles: «Vingt, cent mille ânes dans un pré et cent vingt dans l'autre...»);

- nombres de fantaisie. On a pu glaner deux suites de chiffres, assez ressemblantes, 30-12 et 40-12, renvoyant à des nombres en dehors du système numérique (mais sur l'air connu de 72 et 92), issues probablement du langage des enfants et qui fonctionnent comme des équivalents de trente-six: «Du premier au second degré [d'humour] en passant par le trente-douzième...!» (Gros plan sur Ghostbusters [SOS Fantômes], Paris, Hatier, 1989, livret d'accompagnement de cassette vidéo; présentation par Catherine Terré et Michel Viel) et: «Elle va au secrétariat pour remplir les 'quarante-douze' papelards administratifs que personne ne lira jamais [...]» (E. Hanska, Les Raouls ou la Vie comme au ciné) ou encore, ibid.: «J'ai toujours un tickeçon d'acier avec les vieillards. Dans la galerie du Lido y en a quarante-douze qui me suivent à tête baissée. Quand mon manteau s'ouvre à chaque pas sur mes cuissardes et ma mini-jupe, $z$ 'en paument leurs binocles les pépés.»; 
nombres aléatoires. Il s'agit ici de nombres totalement imprévisibles, fantaisies d'écrivain à partir des chiffres qui leur viennent sous la plume et dont il est sans doute vain de chercher la signification (sont évidemment exclus de cette catégorie les cas en partie prévisibles, notamment ce que l'on appelle couramment les «chiffres ronds»: on les a rangés sous le nombre de base auquel ils peuvent se rattacher, ainsi trois cents ou huit cents sous 100.) On n'en donnera ici qu'un exemple délicieux, tiré d'A. Vialatte qui, avec la dextérité d'un illusionniste, réussit à justifier un nombre aussi arbitraire que 17: «Le camelot vend $L e$ bandit de l'Ohio, l'escroc d'imaginaires terrains de la NouvelleJérusalem 'dans le nord du Massachusetts'. Il en décrit l'embarcadère, les monuments, le jardin botanique. 'Ce petit point sur le plan, dit-il, est une fontaine perpétuelle. Remarquez les dix-sept astérisques : ce sont les lycées qu'on a bâtis.' Dix-sept est déjà bien joli; dixsept est un chiffre inspiré ; on réfute un, on réfute deux, on réfute vingt; mais dix-sept est machiavélique, dix-sept est tellement arbitraire qu'il a l'incroyable du vrai.» (Profitons de l'ornithorynque.) En fait, l'auteur récidivait: il avait déjà mis en scène dans Les Fruits du Congo, avec le même bonheur, 23 «le nombre même du hasard modéré [...] le fait d'un hasard mûri dans la logique en vue d'être le comble efficace du hasard»! D'autres écrivains sont plus prodigues, ainsi A. Hardellet dans Les Chasseurs: «Une foule énorme dansait et ondulait au son de sept cent quatre-vingt-douze mille orgues électriques, illuminée par sept cent quatre-vingt-treize milliards de volts», le ruban bleu provisoire appartenant probablement à J.-L. Benoziglio dans Béno s'en va-t-en guerre: «[...] milliards de milliards de milliards de milliards de milliards de milliards de milliards de milliards de milliards de milliards de milliards de milliards de milliards de possibilités [...]» Mais ce n'est sans doute pas un hasard si milliards se lit treize fois dans ce dernier passage!

\section{Dix-huit! Sept écus!}

On mentionnera également quelques cas particuliers qui eux aussi ont été écartés, et d'abord celui, savamment appelé, de l'homonymisation : rapprochement qui est fait entre le cri d'un oiseau par exemple et un élément de la langue, sans aucune intention signifiante. Ainsi, dix-huit ou vingt-huit traduisant le cri du vanneau : «Au fond de sa musette, il montrait trois vanneaux étendus, bleu et vert, tachés de sang. [...] - Je ne goûterai jamais les dix-huit de cet homme-là, interrompit Alcide. - Les dix-huit? - Oui, c'est le cri du vanneau, tu sais bien.» (P. Vimeru, Chutt le Hutteux), ou encore: «Les vanneaux sont des oiseaux espiègles. Quand, effarouchés, ils reprennent leur essor, ils poussent un cri que les hommes traduisent par: vingt-huit - comme si les oiseaux se souciaient de ces chiffres grossiers devant leur mystérieux appel au voyage.» (C. Forot, Odeurs de forêts.)

Une autre catégorie, admirablement illustrée par les travaux récents de Jean-Pierre Chambon, est celle des démimologismes, c'est-à-dire des noms d'oiseaux tirés d'un mimologisme (formule par laquelle on interprète plaisamment les chants des oiseaux): ainsi, et pour n'en donner qu'un exemple, dans beaucoup de campagnes de France la mésange est appelée «huit-écus» (du dialogue qu'elle est censée nouer au printemps avec sa servante). En voici une variante, qui a l'avantage d'expliciter cette petite mise en scène: «Sept écus! Sept écus! C'est la mésange qui loue sa servante, affirmait papa.» (G. Chevereau, Une enfance à la campagne)

\section{«Le numéro deux se réjouit d'être impair»}

On connaît (ou on peut connaître) le vers de Virgile célébrant les propriétés mystiques que les Anciens attribuaient aux nombres impairs Numero deus impare gaudet («Le nombre impair plaît à la divinité» Bucoliques, 8,75 ) et la traduction réjouissante qu'en aurait ânonnée un potache: «Le numéro deux se réjouit d'être impair»! Sans prétendre entrer ici dans de longues considérations, on fera quelques remarques simples qui peuvent aider à mieux situer ou comprendre un certain nombre des expressions du dictionnaire. 
Le train onze. Le profil graphique du chiffre a pu donner naissance à des mots ou à des expressions, souvent d'origine argotique et qui le sont restées : ainsi le zéro «l'anus» (dit aussi le rond), le quatre de chiffre «piège en forme de quatre», le (numéro) sept «crochet de chiffonnier», le train onze «la marche à pied» (expression bien vivante également au Niger, tandis que le Zaïre parle de la ligne onze, et qui évoque l'image des deux jambes formant le chiffre 11), un soixante-six «un accroche-cœur» (vieilli), etc. Plutôt que d'allonger la liste, on la résumera par cet exemple, fourni par Valmont de Bomare au XVIII siècle : «Elles [les ailes du papillon Vulcain] sont communément chargées vers le milieu de quelques caractères de couleur de bistre foncé, qui, selon quelques amateurs, figurent les chiffres 98 ou 78 ou 67. Ces caractères, ainsi que la diversité de ses nuances, lui ont fait donner beaucoup d'autres noms, [...] le quatre-vingt-dix-neuf, le papillon à numéros [...].» (Dictionnaire raisonné universel d' histoire naturelle, Paris, éd. 1791, t. 1, p. 267.)

On peut accrocher à ce profil graphique tout ce qu'on tire de la position des aiguilles du cadran d'une horloge. Pour s'en tenir à la matière de notre travail, citons à deux heures moins dix, à neuf heures et quart, à huit heures vingt, à dix heures ou à dix heures dix. C'est à cette image du cadran que se rattachent diverses métaphores (mais elles ne semblent pas lexicalisées, à la différence de l'argotique marquer midi), évoquant une virilité plus ou moins marquée et dont on ne citera que deux exemples : «Ce fut Ti-SaintLouis qui s'aperçut le premier qu'au lieu de faire un bon trois-heures de l'après-midi ou même un neuf-heures-le-moins-quart si l'on préfère, il n'obtenait qu'un lamentable sixheures-et-demie.» (E. Pépin, L'Homme au bâton) et: «Quand on voyait défiler Mademoiselle, on remerciait le ciel de ne pas vous avoir fait tortue chez un marchand de peignes ou chamois chez un laveur de bagnoles. On se disait illico que c'était une bonté de la Providence que d'avoir un physique avantageux (comme c'est mon cas), deux bras musclés et le cadran solaire sur quatre heures moins dix (y en a tellement qui l'ont sur une heure et demie !).» (San-Antonio, Du sirop pour les guêpes.)

Le profil phonétique est lui aussi à l'origine d'expressions (le dites 33 du médecin qui ausculte son patient) ou de pirouettes graphiques qui, elles, n'avaient pas leur place dans ce travail, du genre : «Il me rassure pleinement et 13 amicalement et je le quitte confiant.» (R. Fallet, Carnets de jeunesse), et dont on peut rapprocher les rébus mentionnés plus haut et certaines comptines ou chansons enfantines comme l'inusable $Y$ a qu'un ch'veu sur la tête à Mathieu.

- Les mille et une nuits. Dans la vie courante, certaines expressions font penser à la cerise sur le gâteau, comme treize à la douzaine ou le treizième mois. Mais l'hyperbole est une figure fréquemment mise à contribution dans les expressions figurées : elle consiste souvent ici dans l'ajout d'une unité à un ensemble clos $(n+1)$, c'est le cas par exemple de la troisième mi-temps, du mouton à cinq pattes, du huitième et du neuvième art, de la huitième merveille du monde, du vingt-et-unième arrondissement, de la vingt-cinquième heure ou des trente-deux jours par mois. (On trouve le même procédé dans un dicton peu aimable pour les gens d'un village voisin, évoqué par C. Menuet, Une enfance ordinaire : «Villeterreux fendu en deux, quatorze maisons, quinze voleux.») Le procédé inverse $(n+1)$ est plus rare, mais on en trouve cependant des exemples, ainsi la quatre-vingt-dixneuvième fois ou cent six ans.

\section{«À la septième fois, les murailles tombèrent»}

S'agissant de la valeur symbolique des nombres, on sait que certains d'entre eux, variables selon les civilisations, ont été chargés depuis la plus haute antiquité d'une valeur particulière, mais ces symboles n'entrent que pour une faible partie dans les relevés qui suivent. On notera par exemple que si 4 peut évoquer une quantité dérisoire (quatre sous, quatre pelés et un tondu), il a aussi une valeur de complétude dans aux 
quatre vents, les quatre points cardinaux ou dire ses quatre vérités à quelqu'un; si le 5 est pour la Gnose symbole de la quintessence, il est d'abord à la base de plusieurs expressions comme le chiffre correspondant aux doigts de la main (notamment dans être comme les cinq doigts de la main), comme 10 , celui des deux mains, a donné naissance au système décimal; on sait que le 7 revêt dans la Bible une importance toute particulière (les sept jours de la semaine, le chandelier à sept branches, les sept piliers de la Sagesse, les sept années de vaches grasses ou de vaches maigres, etc.) tandis que la tradition chrétienne a retenu par exemple les sept sacrements et les sept péchés capitaux : il n'entre guère toutefois dans des expressions figurées. «Certaines expressions familières eurent-elles aussi une origine maçonnique? On l'a pensé pour en voir trente-six-chandelles, allusion possible à l'illumination subite de l'initiation, mais un doute raisonnable subsiste !» (A. Mellor, La Vie quotidienne de la Franc-maçonnerie française $d u$ XVII ${ }^{e}$ siècle à nos jours, Paris, Hachette, 1973, p. 111.)

A six pieds sous terre. Signalons enfin quelques expressions qui conservent des traces antérieures au système métrique, comme à six pieds sous terre ou encore une tête de six pieds de long.

\section{La mouche à quatre culs}

On n'a pas hésité à enregistrer un nombre appréciable d'expressions (nous deux..., la mouche à quatre culs, le quatre sous) ou de variantes (s'en foutre comme de l'arcaran) caractéristiques de telle ou telle région de France. Elles ont leurs lettres de noblesse, même si les dictionnaires les ignorent, et il est sympathique de voir qu'elles ont (eu) des défenseurs à l'Académie française: Claudel, par exemple, n'hésitait pas à défendre le tour nous deux lui comme un de «ces gallicismes naifs qui sont l'élixir le plus savoureux de notre terroir». Une collecte systématique, attentive aux parlures des différentes régions de France, en eût fait apparaître bien d'autres : ainsi à Marseille porter le chapeau sur les onze heures «sur l'oreille», dans 1'Ouest, manger des quatre coins de la goule «manger goulûment», debout quat' sous! «pour tirer un enfant du lit», sans oublier, dans l'Ouest encore et aussi ailleurs, les nombreuses Marie-trois-chausses «femme sans ordre», Marie-quatre-langues ou Marie-mille-goules «femme bavarde» et Marie-quatre-emballes ou Marie-quatre-bras «femme qui fait l'importante», désignations péjoratives, à vrai dire vieillies, que compléterait le québécois Marie-quatre-poches «femme mal habillée, débraillée».

Les exemples ne manquent pas, en effet, en dehors de France, qu'il s'agisse justement du Québec: battre quatre as «être insurpassable, ne pas avoir son pareil»; n'avoir pas inventé le bouton à quatre trous, équivalent de «n'avoir pas inventé la poudre»; le bonhomme sept heures, équivalent du «marchand de sable»; être/se mettre sur son trente-six «sur son trente et un»; avoir les yeux grands comme des cinquante cennes «ouvrir de grands yeux, des yeux ronds» (comme une pièce de cinquante cents); jamais dans cent ans «jamais de la vie, au grand jamais»; de l'Algérie: «Si [...] vous faites escale en Algérie, vous serez étonné de voir que ce pays, dont les gouvernements vitupèrent la francophonie depuis l'indépendance (1962), n'a en revanche pas pu empêcher dans la rue une vigoureuse créativité parolière en français : dès l'aéroport, votre taxiteur [...] vous entretiendra des 404-bâchées «les femmes voilées» [...] ou des 33-tours «les enturbannés» [...].» (J.-P. Péroncel-Hugoz dans Le Monde, 19 octobre 1991, p. 18, col. 6); ou encore de l'Afrique noire où l'on a relevé: deuxième bureau «maîtresse d'un homme marié» (République centrafricaine, Rwanda, Zaïre); deux doigts «pickpocket» (Côte-d'Ivoire, Mali, Sénégal); homme à quatre yeux: «personne dotée d'un pouvoir surnaturel de communication avec l'au-delà» (Cameroun); quatre fois cinq «vin de palme» 
(par jeu de mots, Sénégal); six lettres «coureur de jupons», les six lettres étant celles du mot bordel, employé en ce sens (Burkina, République centrafricaine, Mali, Tchad); article quinze «système D» (Zaïre). Sans oublier, en achevant ce rapide aperçu par la France des DOM-TOM, le tit' cinq pou cent, punch des Antilles ainsi nommé en souvenir des $5 \%$ de la production accordés autrefois par la Régie à la consommation familiale (pratique équivalente à la part des anges du cognac charentais), ou les bavards impénitents qu'à Saint-Pierre-et-Miquelon on dit avoir une gueule de trente-six.

Si l'on quitte la francophonie, on retrouve bien sûr les mêmes phénomènes dans diverses langues. L'anglais parle, par exemple, du nineteenth hole (le $19^{\mathrm{e}}$ trou du golf) pour désigner le bar où l'on se rafraîchit après le parcours (ou, parfois, un rendez-vous galant); dans la langue enfantine, number one et number two sont les équivalents respectivement de «la petite» et de «la grosse commission»: « [...] Blanchette en profite pour aller dans l'une des ladies rooms aménagées le long des couloirs circulaires de l'arène, s'acquitter du petit 'number one' qui la taraude depuis le début du spectacle.» (L. Chalumeau, Fuck.) De son coté, l'allemand ne dit pas «manger comme quatre» mais «pour trois» (für drei essen); «être bête comme ses pieds» y a pour équivalent littéral «être incapable de compter jusqu'à trois» (nicht bis drei zählen); quelqu'un de haut comme trois pommes bénéficie d'une autre image: «haut comme trois fromages» (Dreikäsehoch), proche de notre ancien «haut comme deux sous de fromage mou»; et les mélomanes savent que L'opéra de quat' sous traduit Die Dreigroschenoper de B. Brecht, sans que le cours du mark y soit pour quelque chose.

PIERRE RÉZEAU 